\title{
Mechanical quality factor measurements of monolithically suspended fused silica test masses of the GEO 600 gravitational wave detector
}

\author{
J R Smith ${ }^{1}$, G Cagnoli ${ }^{2}$, D R M Crooks ${ }^{2}$, M M Fejer ${ }^{3}$, S Goßler ${ }^{1}$, \\ H Lück ${ }^{1}$, S Rowan ${ }^{2,3}$, J Hough $^{2}$ and K Danzmann ${ }^{1}$ \\ ${ }^{1}$ Max-Planck-Institute for Gravitational Physics (Albert-Einstein-Institute) and University of \\ Hannover, Callinstr. 38, D-30167 Hannover, Germany \\ 2 Institute for Gravitational Research, Department of Physics and Astronomy, \\ University of Glasgow, Glasgow G12 8QQ, UK \\ ${ }^{3}$ Edward L Ginzton Laboratory, Stanford University, Stanford, CA 94305-4088, USA \\ E-mail: jrsmith@aei.mpg.de
}

Received 31 August 2003

Published 12 February 2004

Online at stacks.iop.org/CQG/21/S1091 (DOI: 10.1088/0264-9381/21/5/105)

\begin{abstract}
Internal thermal noise is expected to be a limiting noise source in the most sensitive frequency band of the GEO 600 gravitational wave detector. Because thermal noise is directly related to energy dissipation, care has been taken to construct test mass suspensions from low-dissipation materials and to eliminate inter-material rubbing where possible. Recently, the GEO 600 team finished the installation of triple-pendulum suspensions for the optics of the Michelson interferometer. Each of these triple pendulums incorporates a monolithic fused silica pendulum as the lowest stage. We have made internal mode quality factor measurements of three monolithically suspended test masses. Using these measurements we estimate of the level of internal thermal noise in the GEO 600 interferometer.
\end{abstract}

PACS numbers: $04.80 . \mathrm{Nn}, 95.55 . \mathrm{Ym}, 62.40 .+\mathrm{i}$

\section{Introduction}

A worldwide network of interferometric gravitational wave detectors is being assembled [1-4], and most of these detectors have begun taking science data. Scientists are working on these instruments to reduce technical noise sources in order to achieve better sensitivity and thereby increase the probability of detecting gravitational wave events. As the sensitivity of the interferometers increases, they will finally confront fundamental noise sources, inherent in the instruments, which will limit the number of events each can detect. 


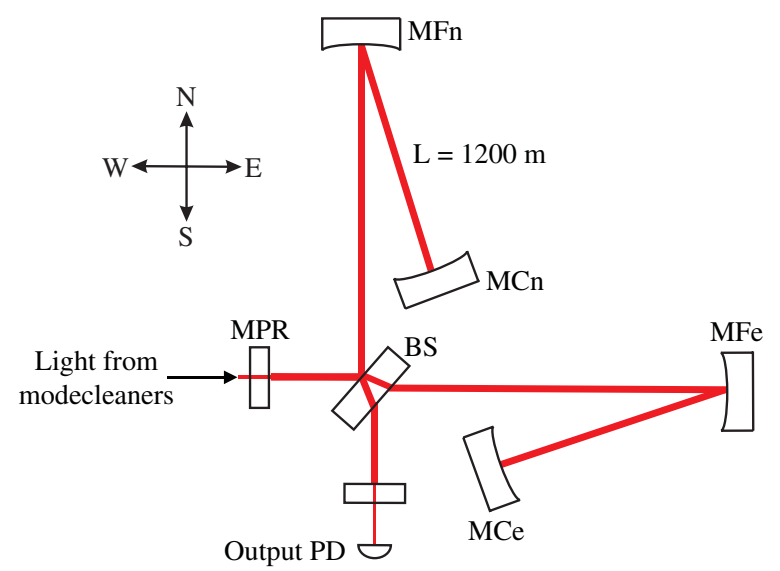

Figure 1. Simplified optical layout of GEO 600. The end mirrors (MCe and MCn), far mirrors (MFe and MFn) and the beamsplitter (BS) are suspended monolithically.

Thermal noise, a fundamental noise source caused by thermally driven fluctuations in the interferometer optics and their suspensions, is expected to be one of the limiting noise sources in GEO 600s most sensitive frequency band [1]. Thermal noise can be reduced by cooling interferometer optics and suspensions to cryogenic temperatures, which requires a high capital investment, and/or by constructing interferometer optics and suspensions from materials with low dissipation and with as few inter-material joints as possible. GEO 600 operates at room temperature. Thus to achieve acceptable thermal noise levels, its test masses and beamsplitter are made from fused silica, a material known to have low mechanical loss [8]. In addition, these optics are suspended monolithically from a fused silica intermediate mass via four fibres flame welded at each end to standoffs (or 'ears') which are attached to the mirror and intermediate mass by hydroxy-catalysis (or 'silicate') bonding [5]. Each of these monolithic fused silica suspensions comprises the lowest stage of a triple pendulum.

Thermal noise contributions to length noise in interferometers are often decomposed into suspension thermal noise, which describes the motion of the test mass surface due to fluctuations in the pendulum, and internal thermal noise, which describes the motion of the test mass surface due to fluctuations within the mirror itself. The monolithic suspensions are expected to have pendulum modes with very low loss in the operational frequency band of GEO 600. For this reason, apart from sharp violin mode resonance peaks, suspension thermal noise is not expected to limit the sensitivity of GEO 600 at any frequency. Internal thermal noise, which is related to the loss factors of the test masses, is expected to be a limiting noise source in GEO600s most sensitive frequency band. In order to create an estimate of the thermal noise limit to strain sensitivity of the GEO 600 detector, we have made loss measurements of three monolithically suspended test masses.

\section{Experiment}

A simplified optical layout of the GEO 600 interferometer is shown in figure 1. The end mirrors (MCe and MCn) and the far mirrors (MFe and MFn) as well as the beamsplitter (BS) are each suspended as the lowest stage of a triple pendulum. The lowest two stages of this pendulum are shown in figure 2. A reaction pendulum is located $3 \mathrm{~mm}$ behind MCe and MCn 


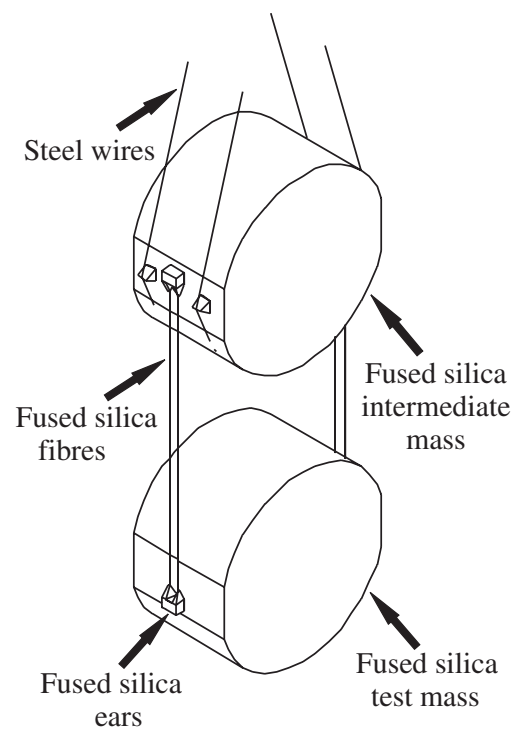

Figure 2. Diagram of a GEO 600 monolithic suspension. The test mass is suspended from the intermediate mass by four fused silica fibres via fused silica ears silicate bonded to the test mass.

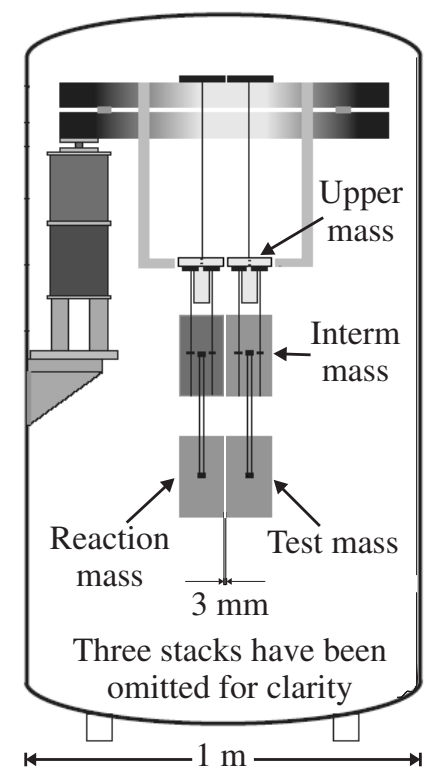

Figure 3. Test mass suspension and reaction pendulum for $\mathrm{MCe}$ and $\mathrm{MCn}$. The electrostatic drive is located on the face of the lowest mass of the reaction pendulum.

to provide a stable reference for applying length control and alignment actuation. The test mass suspension and reaction pendulum are shown in figure 3 and described in [6].

The lowest mass of each reaction pendulum is made of fused silica and has electrodes for an electro-static drive (ESD) deposited on its front surface. The ESDs have four quadrants, each consisting of a comb-capacitor (see [7] for details). In addition, there is a crude ESD, 


\begin{tabular}{|c|c|c|c|c|c|c|c|c|}
\hline Mode: & 7 & 9 & 12 & 17 & 18 & 19 & 28 & 32 \\
\hline Shape: & & & & & & & & \\
\hline Fr. [kHz]: & 11.1 & 15.2 & 17.4 & 19.2 & 19.4 & 19.7 & 25.7 & 26.5 \\
\hline
\end{tabular}

Figure 4. FEA-predicted amplified mode shapes and their approximate measured frequency in kHz. Only the modes for which $Q$ was measured are shown.

consisting of a single copper ring, mounted on a post behind MFn. These three ESDs were the actuators for the measurements described below.

The first step in our experimental procedure was to determine the frequencies of the test mass internal modes. This was done using the finite element analysis (FEA) program Algor. For simplicity the mirrors were modelled as right circular cylinders and the flats, ears and wedge angles were neglected. The measured mode frequencies were all within $1.5 \%$ of the FEA-predicted values, and more typically within 1\%. Some FEA-predicted mode shapes are shown in figure 4 . The FEA mode shapes and frequencies shown are those that most closely matched the frequencies of the modes we measured. A spacial mapping of each measured mode would tell us whether the measured mode shapes correspond to the FEA-predicted shapes, but we have not yet made such a measurement. There were several other modes, such as transverse-stretching modes, that we were unable to detect. This is likely because they did not cause significant longitudinal displacement of the test-mass surface.

The dissipation measurements were carried out by exciting resonant modes of the test masses, then measuring the free decay of the vibrations. The modes were located with bandlimited (typically 50 to $100 \mathrm{~Hz}$ bandwidth) pseudo-random noise or periodic chirps generated using a spectrum analyser and centred around the FEA-predicted mode frequencies. The spectrum analyser signal was amplified with a high voltage amplifier (HVA), and fed to one of the three ESDs mentioned above. Resonant enhancement of the test mass motion by the drive signal caused differential length changes between the two interferometer arms and was thus visible as a peak in the amplitude spectrum of the differential-errorpoint signal (during interferometer lock). After the frequency of a given mode was accurately known, each mode was excited to a significant signal to noise ratio using a fixed-sine signal at the resonant frequency. Finally, the excitation signal was switched off and the decay of the resonance peak was monitored using a spectrum analyser.

The amplitude ringdowns followed the exponential decay law

$$
A(t)=A_{0} \exp \left(\frac{-t}{\tau}\right),
$$

where $A(t)$ is the time-varying amplitude, $A_{0}$ is the initial amplitude and $\tau$ is the ringdown time constant. To determine $\tau$, the natural $\log$ of $A(t)$ was fit with the line $t / \tau+\ln \left(A_{0}\right)$. The quality factor $Q$ was then calculated using the relation

$$
Q=\pi f_{n} \tau
$$

where $f_{n}$ is the frequency of the $n$th mode.

The results of the $Q$ measurements are shown in table 1 along with the standard deviation of $Q$ among different measurements on the same mode and mirror. The highest $Q$ over all measurements and modes was $3.84 \times 10^{6}$ for mode 7 (or the 'butterfly' mode) of MCe. More modes were measured for MCe than MCn because the ESD of MCn produced a factor of 4 less force than that of MCe at the time of these measurements. The ESD behind MFn produced even less force, thus only mode 9 (the 'drum' mode) of that mirror was detected. 
Table 1. Measured mode frequencies and $Q$ s for three GEO 600 test masses and standard deviation among $Q$ for measurements of a given mode and test mass.

\begin{tabular}{lclll}
\hline Mirror & Mode & Frequency $(\mathrm{Hz})$ & $Q$ & $\sigma$ \\
\hline MCe & 7 & 11062 & $3.84 \times 10^{6}$ & $2.50 \times 10^{4}$ \\
& 9 & 15193 & $4.64 \times 10^{5}$ & $4.80 \times 10^{4}$ \\
& 12 & 17350 & $1.18 \times 10^{6}$ & $1.56 \times 10^{4}$ \\
& 17 & 19199 & $4.48 \times 10^{5}$ & $3.22 \times 10^{4}$ \\
& 18 & 19424 & $3.42 \times 10^{6}$ & $6.46 \times 10^{3}$ \\
& 19 & 19665 & $4.63 \times 10^{5}$ & $1.29 \times 10^{5}$ \\
& 28 & 25717 & $9.60 \times 10^{5}$ & $1.95 \times 10^{4}$ \\
& 32 & 26479 & $1.48 \times 10^{5}$ & $5.53 \times 10^{4}$ \\
MCn & 7 & 11057 & $3.96 \times 10^{5}$ & $4.16 \times 10^{4}$ \\
& 9 & 15194 & $8.63 \times 10^{5}$ & $9.95 \times 10^{4}$ \\
& 17 & 19188 & $5.60 \times 10^{5}$ & $1.54 \times 10^{4}$ \\
& 18 & 19421 & $1.81 \times 10^{6}$ & $2.71 \times 10^{3}$ \\
MFn & 9 & 15183 & $7.41 \times 10^{5}$ & $2.28 \times 10^{4}$ \\
\hline
\end{tabular}

\section{Discussion}

\subsection{Loss mechanisms}

The internal loss of a test mass for a given mode can be described as a sum of the losses of its component materials, each scaled by the ratio of energy stored in that material to the total amount of energy stored in the test mass for that mode. For GEO 600 we have

$$
\phi_{\mathrm{TM}}=\phi_{\text {bulk }} \frac{E_{\text {bulk }}}{E_{\text {total }}}+\phi_{\text {ear }} \frac{E_{\text {ear }}}{E_{\text {total }}}+\phi_{\text {coating }} \frac{E_{\text {coating }}}{E_{\text {total }}}+\phi_{\text {bond }} \frac{E_{\text {bond }}}{E_{\text {total }}},
$$

where $\phi_{\text {bulk }}, \phi_{\text {ear }}, \phi_{\text {coating }}$ and $\phi_{\text {bond }}$ are the loss factors of the bulk fused silica, fused silica ears, mirror coating and silicate bonds, respectively, and $E$ represents the energy stored in a given material during modal oscillation. Each loss factor multiplied by its energy ratio can be thought of as an effective loss $\phi_{\text {eff }}$, which would be the loss of the entire test mass if it were only losing energy due to dissipation in that material. The sum of these effective losses then gives the total test mass loss.

The far and end mirrors of GEO 600 are made of Suprasil 1 brand fused silica manufactured by Heraeus Amersil, Inc. Each has a radius of $9 \mathrm{~cm}$ and a thickness of $10 \mathrm{~cm}$. The bulk loss factor of Suprasil 2, a very similar type of fused silica, has been measured to be around $1.8 \times 10^{-8}$ [8]. We take this value as the loss factor of the test mass bulk. Because the bulk comprises almost all of the test mass volume, the energy ratio for the bulk term in equation (3) may be approximated as unity. Thus, the effective loss of the bulk is approximately equal to the loss factor.

Two different types of fused silica ears are used in GEO 600. The first type is $3 \mathrm{~cm}$ long, $1 \mathrm{~cm}$ high and $5 \mathrm{~mm}$ tapering to about $1 \mathrm{~mm}$ in depth. The second type is the same, but with a constant depth of $5 \mathrm{~mm}$. We will consider the second type in the following to give a more conservative estimate of the loss contribution of the ears. The loss factor of the ears should be comparable, but somewhat higher than that of the bulk because they are produced from a lower grade of fused silica. The loss factor of the ears will be further increased by surface losses due to the larger surface to volume ratio of the ears (6.7), when compared to that of 
the bulk (0.42). Nevertheless, because the ears occupy around 1500 times less volume than the bulk, the energy stored in the ears for a given mode will be much less than that stored in the bulk when the mode frequency is far from any resonances of the ear. Thus we consider the effective loss of the ears to be negligible.

If we now make the very inaccurate but helpful assumption that the energy is distributed equally within the test mass volume for a given mode, the effective loss of the silicate bonds can be expressed as a volume ratio,

$$
\begin{aligned}
\phi_{\text {eff bond }} & =\phi_{\text {bond }} \frac{E_{\text {bond }}}{E_{\text {tot }}} \\
& \approx \phi_{\text {bond }} \frac{V_{\text {bond }}}{V_{\text {tot }}} \\
& \approx \phi_{\text {bond }} \frac{A_{\text {bond }} t_{\text {bond }}}{\pi r^{2} t} .
\end{aligned}
$$

For the near and far mirrors we have $6 \mathrm{~cm}^{2}$ as the total bond area $A_{\text {bond }}$ (each test mass has two silicate bonds), and $9 \mathrm{~cm}$ and $10 \mathrm{~cm}$ as the test mass radius $r$ and thickness $t$, respectively. Measurements of similar bonds (i.e., silicate bonds formed in the presence of a sodium silicate solution containing $\mathrm{SiO}_{2}$ ) have shown that the loss of the bond $\phi_{\text {bond }}$ is around from $1.8 \times 10^{-1}$ to $5.4 \times 10^{-1}$ and that its thickness $t_{\text {bond }}$ is around $81 \mathrm{~nm}$ [12]. These values give an effective loss of the bonds of

$$
\phi_{\mathrm{eff} \text { bond }} \approx 4.2 \times 10^{-9} \text { to } 1.3 \times 10^{-8} \text {. }
$$

Again assuming uniform energy distribution, we find that the effective coating loss reduces to a thickness ratio,

$$
\begin{aligned}
\phi_{\text {eff coating }} & =\phi_{\text {coating }} \frac{E_{\text {coating }}}{E_{\text {tot }}} \\
& \approx \phi_{\text {coating }} \frac{V_{\text {coating }}}{V_{\text {tot }}} \\
& \approx \phi_{\text {coating }} \frac{t_{\text {coating }}}{t} .
\end{aligned}
$$

The high-reflection coatings on the surfaces of the test masses consist of 30 alternating layers of silica $\left(\mathrm{Si}_{2} \mathrm{O}_{3}\right)$ and tantala $\left(\mathrm{Ta}_{2} \mathrm{O}_{5}\right)$ each with an optical thickness of $\lambda / 4$. Measurements of similar coatings on fused silica substrates have shown that $\phi_{\text {coating }}$ and $t_{\text {coating }}$ can be expected to be around $2.7 \times 10^{-4}$ and $4.27 \mu \mathrm{m}$, respectively $[13,14]$. Substituting these values into equation (6) gives us

$$
\phi_{\text {eff coating }} \approx 1.2 \times 10^{-8}
$$

For the case of modes 7 and 9, the butterfly and drum modes, the effective loss due to the

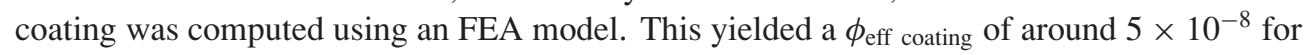
each mode.

Adding each of these effective losses gives an effective total loss of

$$
\phi_{\text {eff }}=\phi_{\text {bulk }}+\phi_{\text {eff bond }}+\phi_{\text {eff coating }}=8 \times 10^{-8},
$$

which is equivalent to a quality factor of around $1 \times 10^{7}$.

There are several drawbacks to the above analysis. Probably the most significant of which is the fact that we assumed uniform energy distribution, while in any realistic mode, energy distribution will be non-uniform. A more thorough analysis can be done using an FEA model to calculate the energy ratios for each mode. Also we have not taken into account loss associated with the superpolished surfaces and barrel polish, but we believe that these losses 
will be smaller than those due to the bonds and coating. We have also neglected energy loss to the intermediate mass, which may accept energy because it is closely matched to the test mass in size, shape and material. We have also, at this stage, ignored losses associated with the fused silica fibres. We will return to this idea below.

Joule damping in the leads after the output of the HVA must also be considered [15]. The ESDs were energized throughout the measurements because they are necessary for length control and alignment of the interferometer. Change in the separation between the test mass and the ESD results in a change in capacitance and thus a current flow. If this current is dissipated in any real impedance of the leads from the HVA output, pendulum damping will occur. For the set-up described here it can be shown [15] that the lower limit on pendulum $Q$ due to this damping is around $10^{9}$. It should also be noted that the feedback loop to the ESDs has a unity gain frequency of around $100 \mathrm{~Hz}$, and thus there is no gain at the frequencies measured here. As an additional check of these statements, we measured $Q$ with the reaction pendulum moved closer to the test mass and observed no change in $Q$.

The variation between the quality factors measured for the different modes of the test masses as shown in table 1 suggests that the measured loss may be strongly affected by energy dissipation in the suspension fibres. This phenomena has been observed in other high $Q$ systems [16]. It is predicted that the fundamental level of thermal noise in a suspended test mass is not affected by the degradation of the measured $Q$ caused by dissipation in the suspension wires [16].

\subsection{GEO 600 internal thermal noise}

To calculate the internal thermal noise we need the substrate loss for each mirror. Based on considerations from the last section and the fact that each of the test masses are made of the same materials, we take the inverse of the highest $Q$ measured over all mirrors and modes as an upper limit on the substrate loss. Thus we have $\phi_{\text {substr }}=2.6 \times 10^{-7}$. In addition, we use the measured beam intensity radius (the distance at which the intensity of the beam goes to $e^{-1}$ of its central value), $r_{0}$ for each test mass. These were $8 \mathrm{~mm}$ for the inboard mirrors and the beamsplitter and $21 \mathrm{~mm}$ for the far mirrors. Neglecting mirror coatings and using Levin's method [10] for predicting thermal noise, with the correction from Thorne and Liu [9] as presented in equation (3) of Nakagawa et al [11] (divided by $(2 k)^{2}$ to get displacement and using $\left.\omega_{0}=\sqrt{2} r_{0}\right)$, we get mirror motion amplitude spectral density

$$
X_{\text {substr }}(f)=\frac{9.40 \times 10^{-20}}{\sqrt{f}} \sqrt{\frac{21 \mathrm{~mm}}{r_{0}}}\left[\mathrm{~m} \mathrm{~Hz}^{-1 / 2}\right] .
$$

Coating thermal noise must also be considered [13,14]. We approximate the coating as a slab of lossy material as in equation (18) of [11]. This gives the thermal noise arising from dissipation in the mirror substrate and coating

$$
X_{\text {GEOTM }}(f)=\frac{1.01 \times 10^{-19}}{\sqrt{f}} \frac{21 \mathrm{~mm}}{r_{0}} \sqrt{\frac{t_{\text {coating }}}{4.27 \mu \mathrm{m}}}\left[\mathrm{m} \mathrm{Hz}^{-1 / 2}\right],
$$

where we have used the coating loss factor and mirror coating thickness given in subsection 3.1 and $r_{0}$ and $\phi_{\text {substr }}$ from above. The beamsplitter has a coating thickness of about $0.5 \mu \mathrm{m}$, and thus will contribute less coating thermal noise than the far and end mirrors.

This estimate for thermal noise neglects contributions from the ears, bonds and other materials that are spatially separated from the mirror surface. We believe this to be a good approximation because experiments by Yamamoto et al [17] have shown that contributions to mirror thermal noise depend strongly on the position of the lossy material with respect to the 
mirror surface. We are currently carrying out further work to take these effects into account in order to make a more accurate estimate of the expected thermal noise level.

GEO 600 is sensitive to a strain

$$
h=\frac{2 \delta L}{L},
$$

where $L$ is the arm length $(1200 \mathrm{~m})$ and $2 \delta L$ is the differential arm length change. As a result of the folded-arm configuration, the beam will bounce twice off each far mirror per round trip. Thus, assuming that all mirrors have the same level of thermal noise, the contribution of thermal noise from MFe and MFn will be more than that from MCe and MCn and the beamsplitter will contribute as much as both end mirrors combined. Taking the contribution of each mirror into account individually we have

$$
h(f)=\frac{2 \sqrt{\frac{1}{4} X_{\mathrm{MCn}}^{2}+\frac{1}{4} X_{\mathrm{MCe}}^{2}+X_{\mathrm{MFn}}^{2}+X_{\mathrm{MFe}}^{2}+\frac{1}{2} X_{\mathrm{BS}}^{2}}}{L},
$$

where $X$ is the amplitude spectral density of longitudinal motion for each mirror. Taking the appropriate values for each mirror from equation (10) gives

$$
h(f)_{\mathrm{GEO}}=\frac{1.16 \times 10^{-21}}{\sqrt{f}}\left[\mathrm{~Hz}^{-1 / 2}\right] .
$$

This level agrees well with previous estimates [1], and should be considered as a preliminary estimate based on measured values.

\section{Acknowledgments}

We thank Gregg Harry for advice pertaining to the experiment, and Valery Mitrofanov and Sergey Lourie for helpful comments regarding test mass losses. We also thank Anja Zimmer for cross checking our FEA modelled mode frequencies. This work was supported by PPARC in the UK and the Albert Einstein Institute in Germany as well as the Universities of Stanford, Glasgow and Hannover.

\section{References}

[1] Willke B et al 2004 Class. Quantum Grav. 21 S417

[2] Sigg D et al 2004 Class. Quantum Grav. 21 S409

[3] Takahashi R et al 2004 Class. Quantum Grav. 21 S697

[4] Acernese F et al 2004 Class. Quantum Grav. 21 S385

[5] Gwo D H 1998 Proc. of SPIE 1363435

[6] Gossler $\mathrm{S}$ et al these proceedings

[7] Grote H 2003 PhD Thesis University of Hannover

[8] Penn S D, Harry G M, Gretarsson A M, Kittelberger S E, Saulson P R, Schiller J J, Smith J R and Swords S O 2001 Rev. Sci. Instrum. 723670

[9] Liu Y T and Thorne K S 2000 Phys. Rev. D 62122002

[10] Levin Y 1998 Phys. Rev. D 57659

[11] Nakagawa N, Gretarsson A M, Gustafson E K and Fejer M M 2002 Phys. Rev. D 65102001

[12] Sneddon P H, Bull S, Cagnoli G, Crooks D R M, Elliffe E J, Faller J E, Fejer M M, Hough J and Rowan S 2003 Class. Quantum Grav. 20 5025-37

[13] Crooks D R M et al 2004 Class. Quantum Grav. 21 S1059

[14] Penn S D et al 2003 Class. Quantum Grav. 202917

[15] Mitrofanov V P, Styazhkina N A and Tokmakov K V 2002 Class. Quantum Grav. 192039

[16] Logan J E, Robertson N A and Hough J 1992 Phys. Lett. A 170352

[17] Yamamoto K, Otsuka S, Ando M, Kawabe K and Tsubono K 2002 Class. Quantum Grav. 191689 